\title{
IMAGES IN MEDICAL PRACTICE
}

\section{Young Boy with Hematemesis}

\author{
SARUPA $^{\mathrm{a}}$, HT HOSSAIN ${ }^{\mathrm{b}}$
}

(J Bangladesh Coll Phys Surg 2018; 36: 179-180)

DOI: http://dx.doi.org/10.3329/jbcps.v36i4.38189

A 20 years old male patient was admitted to the hospital with the complaints of fever for 9 days, vomiting out of blood for 7 days and black tarry stool for 4 days. He gave history of taking NSAID.

On examination patient was normotensive.

Endoscopy showed multiple erosions in the cardia, fundus and antrum of the stomach. No active bleeding was seen.

CT scan of the whole abdomen was performed. In CT scan oral low attenuation contrast medium i.e. water was given to see any high density area within the bowel

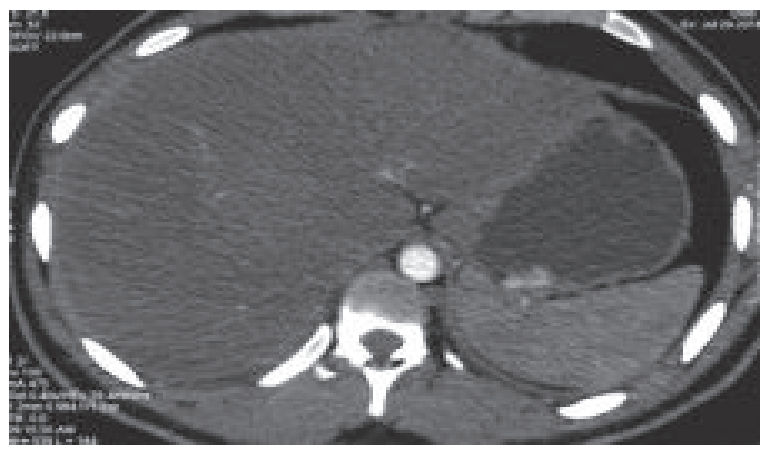

Fig.1 : High density area along fundus of stomach.

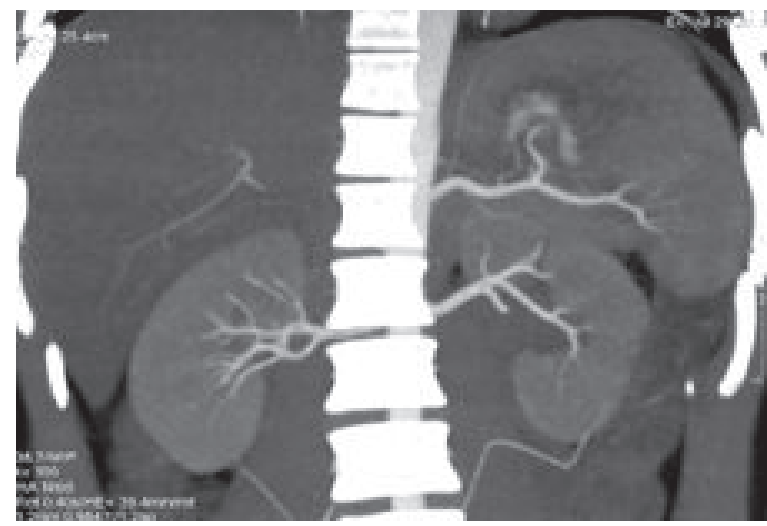

Fig.4: Active bleeding from left gastric artery. to excludeany active bleeding. Twohigh density (70 $\mathrm{HU})$ areas were seen in the fundus and along the lesser curvature of the stomach.

After intravenous contrast administration, early arterial phase i.e. CT angiogram (18 sec after giving I/V contrast) scan was taken to see Aorta and it's branches to identify bleeding vessels. Two active bleeding vessels were identified. One from short gastric artery branch of splenic artery supply the fundus (Fig. 1 and 3) and another from left gastric artery supply the lesser curvature of stomach (Fig. 2 and 4). No aneurysm was identified.

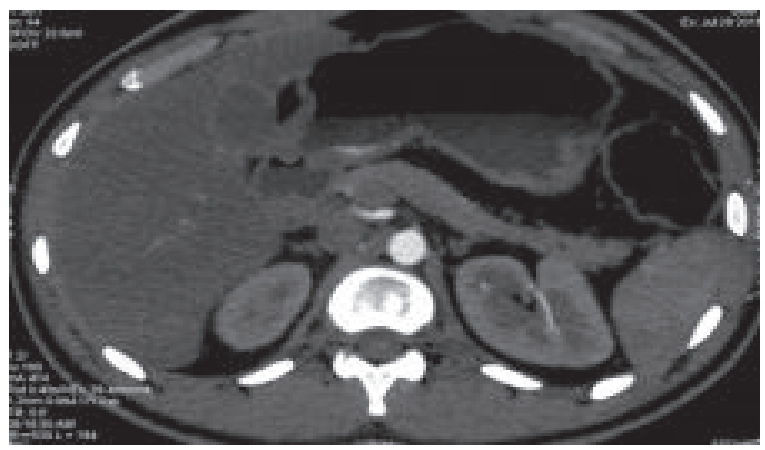

Fig.2 : High density area along lesser curvature of stomach.

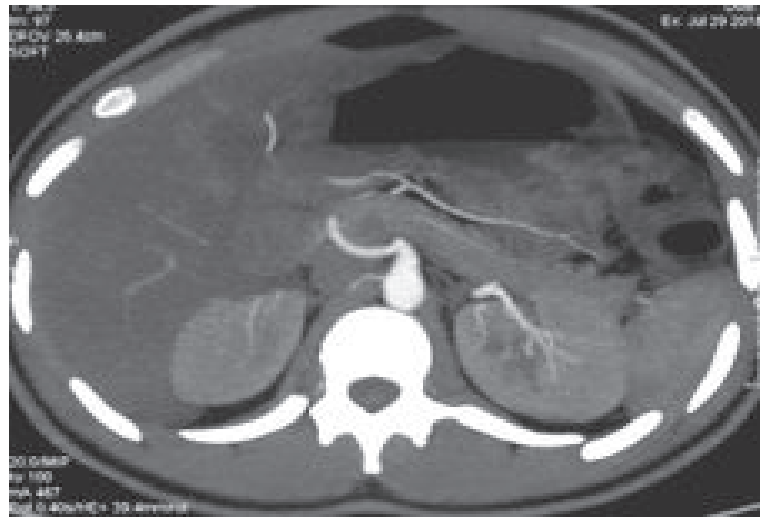

Fig. 3 : Active bleeding from short gastric artery branch from splenic artery.

a. Dr. Sharmin Akhtar Rupa, Associate Professor, Department of Radiology and Imaging, Popular Medical College and Hospital, Dhaka.

b. Dr. HumayraTahseen Hossain, Associate Professor, Department of Medicine, Popular Medical College and Hospital, Dhaka.

Address of correspondence : Dr. Sharmin Akhtar Rupa, Associate Professor, Department of Radiology and Imaging, Popular Medical College and Hospital, Dhaka. 
Finally, Endoscopy and CT scan of whole abdomen including CT angiogram concluded it is a case of NSAID induced erosive gastritis.

\section{References:}

1. Laing CJ, Tobias T, Rosenblum DI, Banker WL, Tseng L, Tamarkin SW. Acute gastrointestinal bleeding: Emerging role of multidetector CT angiography and review of current imaging techniques. Radiographics 2007;27:1055-70.

2. Anthony S, Milburn S, Uberoi R. Multi-detector CT: review of its use in acute GI haemorrhage. ClinRadiat 2007;62:938-49. 